\title{
HIBRIDACIÓN INTERESPECÍFICA EN PASSIFLORA (PASSIFLORACEAE), MEDIANTE POLINIZACIÓN MANUAL, Y CARACTERÍSTICAS FLORALES PARA LA POLINIZACIÓN
}

\author{
WiLliam RAMíREZ B. \\ Profesor Emérito, Facultad de Ciencias Agroalimentarias, Universidad de Costa Rica, \\ San José, Costa Rica. clizano@yahoo.com
}

\begin{abstract}
In the genus Passiflora the evolution of different colors, presence or absence of attractant aromas, corona structure and position with respect to the androgynophore, could have played an important role in speciation and association to very specific and diverse pollinators, such as bees, moths, wasps, hummingbirds, and bats. Passiflora vitifolia (which is pollinated by hummingbirds) produced viable seeds after being manually pollinated with pollen from $P$. ambigua, $P$. edulis, P. platyloba, P. quadrangularis (all of these pollinated by solitary bees), and P. miniata (pollinated by hummingbirds). P. platyloba was able to produce viable seeds after being pollinated with P. miniata. It is here postulated that due to specific pollinators, some Passiflora species have not evolved genetic mechanisms for reproductive isolation, and that new species could have evolved sympatrically through polyploidy.
\end{abstract}

Resumen. En Passiflora, la evolución de diferentes colores, así como la presencia o ausencia de aromas atrayentes, la conformación y la posición de la corona con respecto al androginóforo, pueden haber jugado un papel importante en la abundante especiación y la asociación con polinizadores notablemente específicos y muy disímiles, tales como abejas, polillas, avispas, colibríes y murciélagos. Passiflora vitifolia (polinizada por colibríes) produjo semillas viables cuando fue polinizada manualmente con polen de P. ambigua, $P$. edulis, . platyloba, $P$. quadrangularis (las tres polinizadas por abejas solitarias) y P. miniata (polinizada por colibríes). P. platyloba produjo semillas viables cuando fue polinizada con P. miniata. Se postula que debido a los polinizadores específicos de Passiflora, algunas de las especies no han evolucionado mecanismos genéticos de aislamiento reproductivo y que nuevas especies pudieron haber evolucionado simpátricamente por poliploidía.

Palabras clave / Key words: Passiflora, hibridación interespecífica / interspecific hybridization, polinización / pollination

\section{INTRODUCCIÓN}

Las Pasifloráceas constituyen un grupo con muchas especies y amplia distribución geográfica. Se conocen 18 géneros y cerca de 630 especies (Vanderplank 1996), que se encuentran en estado silvestre en Sudamérica, Centroamérica, Norteamérica, las Antillas, Islas Galápagos, Australia, Filipinas, Asia y muchas islas del Océano Pacífico (Vanderplank 1996). África no tiene especies endémicas. Algunas especies de Passiflora son de valor comercial, pero todas son importantes en la alimentación de animales silvestres, porque producen néctar, polen, frutos, semillas y tejido vegetal para insectos herbívoros. La mayoría de las especies de Passiflora son plantas trepadoras leñosas y perennes; algunas (por ej., Passiflora tica) son arbustivas, lo que puede favorecer la evolución de nuevas especies por hibridación. Souza et al. (2004) informan que muchas especies silvestres de Passiflora presentan características de interés para programas de mejoramiento, pero que la hibridación con especies cultivadas a veces es posible.

Lasfloressonoligolécticasygeneralmentepolinizadas por abejas hembras solitarias; por ej., Centris, Eulaema, Euglossa, Euplusia, Ptiloglossa, Xylocopa, Bombus (Janzen 1968, León 1987) y las abejas sociales Melipona según MacGregor (1976); Epicharis spp. es un visitador frecuente. Algunas especies de Passiflora son polinizadas por colibríes (Knuth 1905, Janzen 1968), mientras que unas pocas son polinizadas por murciélagos, por ej. P. mucronata (Sazima \& Sazima 1978). La polinización por lepidópteros nocturnos no es común. MacDougal (1994) informa que P. hahnii (E. Fourn.) Mast. es visitada por polillas y que otras son polinizadas por avispas. Según Grant (1963), plantas genéticamente emparentadas con frecuencia se diferencian en la estructura, aromas atrayentes y colores de las flores y estas diferencias estructurales y fisiológicas previenen la polinización cruzada entre 
especies. MacDougal (1994) sugirió que el ancestro del género Passiflora era polinizado por insectos, probablemente de Hymenoptera, y que la polinización por colibríes apareció independientemente en varias líneas.

La tendencia en Passiflora está a favor de la polinización cruzada (Knight \& Winters 1962). Janzen (1968) infirió que esto ocurre como consecuencia de la organización de las partes florales. De acuerdo con Vanderplank (1996), el polen de la mayoría de las especies madura hasta el mediodía, pero el estigma es receptivo desde que se abre la flor. Passiflora vitifolia, una de las especies usadas en este trabajo en los cruzamientos, es autoincompatible (East 1940, Snow 1982).

Según Janzen (1968) y Sazima \& Sazima (1978) la flor característica de Passiflora tiene un mecanismo que promueve la polinización cruzada por medio del movimiento alterno de las anteras y los estigmas. Master (1871) describió brevemente el comportamiento floral de Passiflora en contra de la autopolinización. Sazima \& Sazima (1978) informan que al abrirse las flores de $P$. mucronata, las superficies estigmáticas son opacas y sin ninguna secreción, pero al final del movimiento de las anteras y los estigmas, éstos están cubiertos por una secreción pegajosa. Amela-García \& Hoc (1998) informan que en $P$. foetida los estigmas están receptivos durante toda la antesis.

El género Passiflora, así como otras plantas que generalmente tienen polinización cruzada, posee características morfológicas y fisiológicas que la promocionan o demandan; por ej., heterostilia, autoincompatibilidad y protandria (Baker \& Hurd 1968), movimiento alterno de anteras y estigmas, así como presencia o ausencia de aromas atrayentes, diferentes colores y exposición o no del androginóforo (obs. pers.). Vanderplank (1996) menciona la obtención de varios híbridos de Passiflora por polinización manual, algunos de los cuales se han convertido en plantas de interés comercial o en invasoras. Tanto Corner (1958) como Ramírez-B. (1986) indican que no existen híbridos naturales en Ficus; y el autor desconoce informes sobre híbridos naturales en Passifloraceae. La mayoría de las especies de Passiflora son diploides, con $2 \mathrm{n}=12,2 \mathrm{n}=18$ ó $2 \mathrm{n}=20$ cromosomas; también se conocen tetraploides, hexaploides y octaploides (Snow \& MacDougal 1993). El número ancestral de cromosomas en el genéro Passiflora es n $=9$ (Hansen et al. 2006).

Los géneros Passiflora y Ficus (Moraceae, los higos) se caracterizan por poseer muchas especies y amplia distribución geográfica en los trópicos y subtrópicos (630 y 750 especies, respectivamente). Ambos grupos poseen polinizadores específicos. Ramírez-B. (1970) postuló que había una relación causal entre el mecanismo de polinización específica en Ficus y el extraordinario número de especies conocidas y que las especies de Ficus carecían de barreras de aislamiento genético o barreras de incompatibilidad. La ausencia de estas barreras fue indirectamente comprobada por la obtención artificial de híbridos interespecíficos, que produjeron semillas viables en la primera y en la segunda generación (Ramírez-B. 1986); por ej., Ficus glabrata Kunth x F. maxima Mill.

El objetivo de este estudio fue hacer manualmente polinización cruzada entre especies de Passiflora nativas del Nuevo Mundo y determinar las características florales asociadas con sus polinizadores.

\section{Materiales y MÉTOdos}

Flores de Pasiflora vitifolia Kunth se polinizaron manualmente con polen de P. adenopoda D.C., P. ambigua Hemsl., P. miniata Vanderplank ${ }^{1}$, P. edulis Sims f. flavicarpa Deg., P. platyloba Killip, P. quadrangularis L., P. quinquangularis S. Calderón ex J.M. MacDougal y P. tica Gómez-Laur. \& L.D. Gómez. Passiflora platyloba fue polinizada con P. tica, P. miniata y $P$. vitifolia. Las dos últimas especies son autoincompatibles y polinizadas por colibríes. De cada especie polinizada manualmente se usó un máximo de cinco flores.

Las plantas de $P$. miniata, $P$. adenopoda y $P$. vitifolia crecieron a partir de esquejes en la propiedad del autor en Santo Tomás de Santo Domingo, Heredia, Costa Rica, mientras que las plantas de P. ambigua, $P$. quadrangularis y $P$. quinquangularis crecieron en los jardines del Instituto Nacional de Biodiversidad (INBio), Santo Domingo, Heredia. P. edulis f. flavicarpa creció en una casa localizada en Santo Domingo de Heredia, al igual que en el INBio. Las flores de $P$. tica se recolectaron en la Reserva Biológica Alberto M. Brenes, de la Universidad de Costa Rica, situada en la Cordillera de Tilarán. No se observó ninguna otra especie de Passiflora en los alrededores inmediatos al experimento.

\footnotetext{
${ }^{1}$ Según Vanderplank (2006) lo que erróneamente se menciona como P. coccinea Aubl. en realidad corresponde a P. miniata.
} 
A las flores usadas como madres se les eliminó la corona y los estambres antes de su apertura y se aislaron en bolsas plásticas. La polinización fue manual por el contacto de los estigmas con las anteras de la especie correspondiente a cruzar. Las cubiertas de las flores se quitaron un día después de la polinización. La polinización se hizo generalmente antes de las 9 a.m. y en algunos casos antes de las 6 a.m., una hora antes de la apertura de las flores. Los frutos producidos no fueron protegidos. Las semillas provenientes de los cruces se sembraron en macetas plásticas con suelo, dos días después de su secamiento al aire libre.

\section{Resultados}

Producción de frutos y semillas y germinación

Passiflora vitifolia produjo frutos (Fig. 1) y semillas viables cuando fue polinizada manualmente con polen de $P$. ambigua, P. edulis, P. platyloba, $P$. quadrangularis y $P$. miniata (Cuadro 1). Los frutos desarrollaron semillas normales (Fig. 2) y un cruce de P. vitifolia $\mathrm{x}$ P. miniata tuvo 204 semillas maduras. Las flores de esas especies autopolinizadas manualmente, excepto P. platyloba, no produjeron frutos. P. platyloba polinizada con $P$. miniata produjo semillas viables. Passiflora adenopoda y P. quinquangularis $\mathrm{x}$ $P$. vitifolia no produjeron frutos. Flores de $P$. vitifolia y P. platyloba polinizadas por $P$. tica no produjeron frutos (Cuadros 1 y 2). Veinticinco semillas de la F1 del cruce de $P$. vitifolia $\mathrm{x} P$. ambigua germinaron $100 \%$ (Fig. 3); sin embargo, no hubo germinación de 25 semillas obtenidas de la autopolinizacion de $P$. vitifolia. Flores de $P$. vitifolia polinizadas con polen de P. platyloba antes de la antesis (6 a.m.) desarrollaron frutos normales. No se observó en $P$. vitifolia ni en $P$. platyloba que los estigmas se doblaran más abajo de la posición de las anteras, como observó Janzen (1968) en $P$. foetida. Los estigmas de $P$. vitifolia son receptivos desde antes de la antesis, permanecen así durante el día y regresan a la posición vertical al día siguiente de la polinización. Brácteas, sépalos, pétalos y corona de $P$. vitifolia y $P$. platyloba se flexionan hacia el ginóforo durante la noche, después de la polinización, para proteger el ovario fecundado. Las flores de $P$. vitifolia, P. miniata y P. platyloba se cierran al oscurecer, al final del día. En el cruce de P. vitifolia x P. platyloba las cinco flores polinizadas produjeron frutos normales.
Polinizadores y otros insectos asociados con las especies de Passiflora estudiadas

Las flores de Passiflora vitifolia fueron destruidas en la base por Trigona corvina para robar néctar antes de la antesis, como también fue observado por Gill et al. (1982) con dos especies de Trigona y por Sazima \& Sazima (1989) con T. snipites en la granadilla comercial (P. edulis). Trigona corvina también recolecta polen, aunque no poliniza. No se observaron abejas melíferas (Apis mellifera) visitando las flores de las Passiflora estudiadas, aunque el autor posee colonias de abejas africanizadas a menos de $20 \mathrm{~m}$ de distancia. Epicharis lunata y Xylocopa sp. (Anthophoridae), así como Eulaema cingulata (Apidae), visitaron las flores de $P$. platyloba.Eulaema cingulata y una especie de Scoliidae (Fig. 7) visitaron las flores de P. edulis f. flavicarpa. Xylocopa sp. también visitó las flores de esa especie (Fig. 6). El colibrí Amazilia tzacatl visitó solamente las flores de P. vitifolia. Passiflora adenopoda, P. platyloba y $P$. quinquangularis produjeron abundantes frutos por autopolinización natural. P. edulis f. flavicarpa y $P$. quadrangularis produjeron ocasionalmente algunos frutos por polinización natural, mientras que $P$. platyloba produjo frutos cuando fue autopolinizada manualmente. Los frutos de P. platyloba y P. vitifolia maduraron de color verde.

Se observó que las anteras de las Passiflora estudiadas giran sobre su base al ser empujadas manualmente. Las anteras de $P$. vitifolia aceptaron hasta ocho giros seguidos de 360 grados, a favor y en contra del sentido de las agujas del reloj. Estos movimientos giratorios posiblemente favorecen la deposición del polen, tanto en insectos como en vertebrados polinizadores. No se observó que las anteras se inclinaran más abajo de la posición de los estigmas.

\section{Discusión}

Passiflora vitifolia, que es polinizada naturalmente por colibríes, produjo semillas fértiles cuando fue cruzada manualmente con polen de cuatro especies, que naturalmente son polinizadas por abejas y avispas solitarias; también produjo semillas fértiles cuando fue polinizada con $P$. miniata, que es polinizada por colibríes. P. platyloba produjo semillas fértiles cuando fue polinizada con $P$. miniata.

Autoincompatibilidad de las especies estudiadas

Las especies de Passiflora estudiadas, especialmente P. miniata y $P$. vitifolia (polinizadas por colibríes), son autoincompatibles y su morfología y fisiología están 
a favor de la polinización cruzada. Janzen (1968) afirmó que "la flor característica de las pasifloras tiene un mecanismo que promueve la polinización cruzada por medio del movimiento alterno de las anteras y los estigmas". Sin embargo, se cuestiona si esta hipótesis funciona para todas las especies de Passiflora, pues aunque la mayoría de las especies presentan movimientos diferenciales de estas estructuras, además son generalmente autoestériles. Sin embargo, Janzen (1968) supuso que en Passiflora el atraso en la deflexión de los estigmas constituye una ventaja selectiva para la polinización cruzada, con base en la idea de que "si los estigmas se flexionan cuando la flor abre, la mayoría del polen en un estigma vendría de las anteras inmediatas".

La autoincompatibilidad de algunas de las Passiflora estudiadas puede estar dada no sólo por el movimiento diferente de anteras y estigmas, como argumentó Janzen (1968), sino también por la probable incompatibilidad de los granos de polen en los estigmas de la propia planta, debido a constituciones genéticas particulares (Baker \& Hurd 1968). Según León (1987), ciertas plantas de $P$. edulis pueden ser completamente autoincompatibles debido a mecanismos que inhiben el crecimiento del tubo polínico en los estigmas; en otras, la autoincompatibilidad se presenta en grados distintos. El género Asclepias (Asclepiadaceae), con polinizadores muy específicos y con complejos mecanismos florales de aislamiento mecánico (Stebbins 1950), no produjo semillas viables cuando las flores fueron autopolinizadas artificialmente (Macior 1965); esto sugiere la presencia de barreras fisiológicas contra la autorreproducción entre las plantas de la población. Además, de acuerdo a Macior (1965) la infrecuente incidencia de híbridos naturales o artificiales en Asclepias, mencionado por Woodson (1954), puede indicar que existen barreras fisiológicas entre especies. Wyatt \& Broyles (1994) también mencionan que la mayoría de Asclepias son genéticamente autoincompatibles.

Factores biológicos que mantienen la incompatibilidad

Baker \& Hurd (1968) informan que debido a las constituciones genéticas particulares se necesita el intercambio de polen entre plantas. Es probable que las especies de Passiflora que se cruzaron exitosamente con otras especies posean el mismo número de cromosomas. Souza et al. (2004) indican que $P$. edulis y $P$. quadrangularis, así como cuatro especies silvestres de Brasil, poseen $2 \mathrm{n}=18$ cromosomas. La polinización cruzada o la hibridación entre diferentes especies de Passiflora puede haber contribuido a la evolución de nuevas especies por poliploidía. Goldman (2003) señala que "las pasifloras poliploides tienen una distribución más amplia y se encuentran en hábitats más extremos que sus parientes diploides ..." y que "la hibridación ha jugado un papel importante en la evolución de las plantas, no sólo como una nueva fuente de combinación de genes, sino también como un mecanismo de especiación". Según Melo et al. (2001) la amplia presencia de poliploidía (en Passifloraceae) puede deberse a líneas independientes de poliploidía.

\section{Síndromes florales para la polinización}

Entre las características florales importantes en las especies de Passiflora polinizadas por abejas tenemos colores en la gama de los azules, morados, rosados y amarillos, coronas prominentes, androginóforo corto, no expuesto y protegido por la corona (Fig. 4A y Fig. $5 \mathrm{~A}$ ), hipantio aplanado en forma de copa, aromas fuertes, flores péndulas con pedicelo recto, corto y flexible. Las polinizadas por colibríes son de colores rojo-escarlata, morado o rosado (MacDougal 1994), tienen corona corta, no prominente, generalmente blancuzca, androginóforo largo, pedicelo corto (Fig. 4B y Fig. 5B), no flexible, y flores no péndulas. Según Godman $(1994,2003)$ estas flores poseen hipantio alargado. Otras especies que poseen las características mencionadas son P. manicata y $P$. antioquensis; la última posee pedicelo largo y delgado (Vanderplank 1996). Las polinizadas por murciélagos son de color blanco o crema, con corona corta, amarilla en P. tica, no prominente, androginóforo expuesto (Sazima \& Sazima 1978), aroma presente, pedicelo largo, no flexible, flores no péndulas o con pedúnculo péndulo, pero flores en posición erecta (por ej., $P$. tica, obs. pers.). Para otros detalles florales ver el Cuadro 3. El comportamiento de las abejas hembras, polinizadoras de Passiflora, está dado por el olor y el estímulo visual; en las especies polinizadas por colibríes está dado por el color rojo o escarlata de las flores. Passiflora tica posee características florales para la polinización por murciélagos.

En Passiflora la evolución de diferentes colores, el tamaño de las flores, la presencia o ausencia de aromas atrayentes, la conformación del hipantio, la posición de la corona con respecto al androginóforo (Figs. 4 y 5), la orientación gravitacional de las flores, así como la antesis diurna o nocturna, pueden haber jugado un papel importante en la abundante especiación y la asociación con polinizadores oligolépticos, muy 
específicos y tan disímiles como abejas, avispas, insectos nocturnos, colibríes y murciélagos. Baker \& Hurd (1968) anotan que el tipo de constancia floral por parte del insecto antófilo más abundante reduce la posibilidad de polinización interespecífica y que tal mecanismo de aislamiento puede también jugar un papel en la evolución de un nuevo taxon de plantas. Dodson et al. (1969) indican que la atracción específica de polinizadores es característica de las especies de orquídeas más evolucionadas y que en esas plantas los agentes polinizadores aíslan y previenen la hibridación entre poblaciones compatibles. La probable falta de aislamiento genético en algunas especies de Passiflora puede explicar los híbridos interespecíficos obtenidos en este trabajo. En las especies polinizadas por murciélagos, la posición erecta de las flores, los sépalos y pétalos flexionados hacia abajo, la corona reducida y el androginóforo largo y expuesto hacen suponer que la quiropterofilia evolucionó a partir de la ornitofilia. La reducción de la corona en las especies polinizadas por colibríes y posiblemente en las polinizadas por murciélagos se debe, probablemente, a que en las especies polinizadas por abejas la corona constituye el origen principal de los olores atrayentes, como fue observado por Morren (1842, citado por MacDougal 1994).

\section{Implicaciones evolutivas}

Una pequeña población de Passiflora, en la que haya cambiado el número de cromosomas por hibridación, $\mathrm{u}$ otras causas naturales, puede actuar como una isla biológica. Si esta población se asocia con uno o pocos polinizadores específicos, puede dar origen a una nueva especie sin necesidad de aislamiento geográfico, como fue postulado por el autor sobre el género Ficus (Ramírez-B. 1970). La posible evolución de especies isopátricas en Passifloraceae puede explicar, en parte, el gran número de especies conocidas. Sin embargo, Goldman (2003) considera que los híbridos están frecuentemente asociados con una perturbación del hábitat y que, una vez aisladas dos especies, pueden ser eliminadas por disturbios naturales y que el fenómeno de hibridación puede, en parte, ser responsable de la capacidad adaptativa y de la amplia distribución de Passiflora pallida y P. suberosa, mientras que Reiseberg \& Brouillet (1994) argumentan que las especies derivadas por especiación local seguirán una secuencia de polifilia, parafilia y monofilia. En Passiflora, así como en Ficus y probablemente en otros grupos de plantas (por ej., orquídeas superiores, ver Dobzhansky 1951), con polinizadores específicos, las barreras que evitan el intercambio interespecífico de genes no son genéticas y son efectivas en fases previas a la polinización. Esas barreras son producidas por la morfología y la fisiología floral, así como por la respuesta de los polinizadores a esas características fenotípicas. Consecuentemente, las especies están sujetas a mecanismos de aislamiento externos o a mecanismos estructurales y etológicos, no a aislamiento reproductivo. Sin embargo, Dobzhansky (1951) considera que el aislamiento reproductivo es una característica de las especies. La longitud del androginóforo y su posición respecto a la corona, la conformación y posición del opérculo y la profundidad del nectario provocan un aislamiento mecánico, mientras que la ausencia de aromas u olores y la conformación de la flor provocan aislamiento etológico. Dodson et al. (1969) señalan que las modificaciones estructurales de las orquídeas llegan a ser críticas en especies en las que no se excluye uno o pocos polinizadores. Esto sucede cuando esas modificaciones son necesarias para mantener la integridad de especies muy relacionadas e interfértiles, las cuales viven juntas. Maciur (1965) menciona la presencia de especies de Asclepias en la misma flora local.

\section{Consideraciones finales}

Contrario a la idea de Janzen (1968), el movimiento tardío de los estigmas en Passiflora puede ser un mecanismo para evitar que se bloqueen con el polen de la misma flor, así como para economizar polen que podría ser usado en otra planta, al ser transportado por los vectores (obs. pers.). Jersáková et al. (2005) afirman que la autopolinización tiene consecuencias en la adaptación ("fitness") de la plantas, porque reduce el transporte de polen y menos óvulos son exitosamente fertilizados por polen que proviene de otra planta. Snow (1982) observó que en $P$. vitifolia las flores a las que se les eliminaron las anteras dieron origen a más semillas que las flores con anteras intactas. En muchas orquídeas la producción de semillas es alta cuando se hace polinización cruzada manualmente (Guevara et al. 2003).

En Passiflora, así como en Ficus, los híbridos naturales son poco comunes, lo que aparentemente también sucede en las orquídeas con polinizadores específicos. Corner (1958) considera que la ausencia de hibridación natural en Ficus prueba la especificidad de sus avispas polinizadoras. Dressler (1968) indica que la escasez de híbridos interespecíficos en Stanhopea (Orchidaceae) sugiere que ciertas barreras mecánicas, $\mathrm{u}$ otras, son parcialmente efectivas, mientras que 
Gerlach (2002) anota que las orquídeas son fáciles de hibridizar manualmente.

La producción de híbridos interespecíficos en Passiflora por polinización manual puede contribuir al desarrollo de plantas con frutos de mejor calidad comercial o industrial, otras con flores más llamativas como ornamentales, así como plantas para uso medicinal.

Cuadro 1. Polinización cruzada de Passiflora vitifolia* con otras especies.

\begin{tabular}{|l|c|c|c|}
\hline & P. vitifolia* & & \\
\hline & Hibridación & Polinización & Germinación \\
\hline P. adenopoda & - & abejas & - \\
\hline P. ambigua & + & abejas & + \\
\hline P. edulis & + & abejas & + \\
\hline P. miniata & + & colibríes & + \\
\hline P. platyloba & + & abejas & + \\
\hline P. quadrangularis & + & abejas & + \\
\hline P. quinquangularis & - & ¿abejas? & - \\
\hline P. tica & - & ¿murciélagos? & - \\
\hline
\end{tabular}

* P. vitifolia es polinizada por colibríes.

Cuadro 2. Polinización de Passiflora platyloba* con otras especies.

\begin{tabular}{|l|c|c|c|}
\hline & P. platyloba & & \\
\hline & Hibridación & Polinización & Germinación \\
\hline P. miniata & + & colibríes & + \\
\hline P. tica & - & imurciélagos? & - \\
\hline
\end{tabular}

* P. platyloba es polinizada por abejas solitarias pequeñas.

Cuadro 3. Síndromes florales para el diagnóstico de los polinizadores de Passiflora.

\begin{tabular}{|c|c|c|c|}
\hline Síndromes & Abejas & Colibríes & Murciélagos \\
\hline Colores & $\begin{array}{c}\text { azul, morado, rosado, } \\
\text { amarillo }\end{array}$ & escarlata o rojo & blanco o crema \\
\hline Antesis & diurna & diurna & nocturna \\
\hline Androginóforo & corto, no proyectado & largo, proyectado & largo, proyectado \\
\hline Fragancia & intensa & ausente & débil \\
\hline Filamentos de corona & $\begin{array}{c}\text { libres, largos, no } \\
\text { adjuntos }\end{array}$ & $\begin{array}{c}\text { cortos, adjuntos, contra el } \\
\text { androginóforo }\end{array}$ & $\begin{array}{c}\text { cortos, adjuntos, contra el } \\
\text { androginóforo }\end{array}$ \\
\hline Opérculo & $\begin{array}{c}\text { membranoso, } \\
\text { ascendente, plicado }\end{array}$ & $\begin{array}{c}\text { fimbriado, descendente, } \\
\text { adnato }\end{array}$ & $\begin{array}{c}\text { fimbriado, descendente, } \\
\text { adnato }\end{array}$ \\
\hline Pedicelo & corto, flexible, colgante & $\begin{array}{c}\text { largo, no flexible, erecto o } \\
\text { péndulo }\end{array}$ & $\begin{array}{c}\text { largo, no flexible*, erecto o } \\
\text { péndulo }\end{array}$ \\
\hline Tamaño de corona & $\begin{array}{c}\text { tres coronas largas, } \\
\text { libres }\end{array}$ & dos o tres coronas cortas & dos o tres coronas cortas \\
\hline Posición de flores & no salidas del follaje & salidas del follaje & salidas del follaje \\
\hline
\end{tabular}

*P. tica posee pedicelos péndulos, con flores múltiples terminales en posición vertical debido a la curvatura terminal de los pedicelos. 
Agradecimientos. A Armando Estrada Chavarría y Jorge Gómez Laurito por la identificación de las especies de Passiflora estudiadas, a Ingrid Aguilar Monge por la identificación de Trigona corvina, a Paul Hanson por la identificación de las abejas polinizadoras, a la Biblioteca de la Organización para Estudios Tropicales (OET) por la utilización de sus recursos bibliográficos, a Gilbert Fuentes González por sus comentarios, obtención de literatura y edición del documento, y a Ana Cecilia Jinesta León por las fotografías.

\section{LITERATURA CITADA}

Akamine, E.K. \& G. Girolami. 1957. Problems in fruit set in yellow passion fruit.Hawaii Farm Scie. 5: 3-5.

Amela-García, M.T. \& P.S. Hoc. 1998. Biología floral de Passiflora foetida (Passifloraceae). Rev. Biol. Trop. 46(2): 191-202.

Baker, H.G. \& P.D. Hurd Jr. 1968. Intrafloral ecology. Annu. Rev. Entomol. 13: 385-414.

Corner, E.J.H. 1958. An introduction to the distribution of Ficus. Reinwardtia 4(3):325-355.

Dobzhansky, T.G. 1951. Genetics and the origin of species. Columbia Univ. Press, New York. 364 p.

Dodson, C.H., R.L. Dressler, H.G. Hills, R.M. Adams \& N.H. Williams. 1969. Biologically active compounds in orchid fragrances. Science 164: 1243-1249.

Dressler, R.L. 1968. Pollination by Euglossine bees. Evolution 22: 202-204.

East, E.M. 1940. The distribution of self-sterility in the flowering plants. Proc. Amer. Philos. Soc. 82(4): 449518.

Gerlach, G. 2003. La subtribu Stanhopeinae, sus notables mecanismos de polinización, la química de los aromas florales e implicaciones en sistemática. Lankesteriana 7:104-106.

Gill, F.B., A.L. Mack \& R.T. Ray. 1982. Competition between hermit hummingbirds Phaethorninae and insects for nectar in a Costa Rican rain forest. The Ibis 124(1): 44-49.

Goldman, D.H. 2003. Two species of Passiflora (Passifloraceae) in the Sonoran desert and vicinity: a new taxonomic combination and an introduced species in Arizona. Madroño 50(4): 243-264.

Grant, V. 1963. The origin of adaptations. Columbia Univ. Press, New York. 606 p.

Guevara R.E., M.M. Agosto-Pedroza \& R.L. Tremblay. 2003. Effect of flower age on pollination success in Lepanthes sanguinea. Lankesteriana 7: 107-108.

Hansen, A.K., L.E. Gilberth, B.B. Symson, S.R. Downie, A.C. Cervi \& R.K. Janzen. 2006. Phylogenic relationships and chromosome number evolution in Passiflora. Syst. Bot. 31(1): 138-150.

Janzen, D.H. 1968. Reproductive behavior in the
Passifloraceae and some of its pollinators in Central America. Behaviour 32(1/3): 33-48.

Jersáková, J., S.D. Johnson \& P. Kindlmann. 2006. Mechanisms and evolution of deceptive pollination in orchids. Biol. Rev Camb. Philos. Soc. 81(2): 219-235.

Knight, R.J. Jr. \& H.F. Winters. 1962. Pollination and fruit set of Yellow Passion fruit in Southern Florida. Proc. Florida St. Hort. Soc. 75: 412-418.

Knuth, P. 1905. Handbuch der Blütenbiologie; unter Zugrundelegung von Hermann Müllers Werk "Die Befruchtung der Blumen durch Insekten", bearb. von Dr. Paul Knuth. W. Engelmann, Leipzig. 479 p.

León, J. 1987. Botánica de los cultivos tropicales. IICA, San José. p. 403.

MacDougal, J.M. 1994. A revision of Passiflora subgenus Decaloba, section Pseudodysosmia (Passifloraceae). Syst. Bot. Monogr. 41: 1-146.

Macior, L.W. 1965. Insect adaptation and behavior in Asclepias pollination. Bull. Torrey Bot. Club 92(2): 114-126.

Master, M.T. 1871. Contributions to the natural history of the Passifloraceae. Trans. Linnean Soc. London 27: 593-645.

McGregor, S.E. 1976. Insect pollination of cultivated plants. ARS / U.S. Dept. Agric. Handbook No. 496, Washington. $411 \mathrm{p}$.

Melo, N.F., A.C. Cervi \& M. Guerra. 2001. Karyology and ecytotaxonomy of the genus Passiflora L. (Passifloraceae). Plant Syst. Evol. 226: 69-84.

Morren, C. 1842. Recherches litteraires sur les fleurs de la passion, suivies d'observations an anatomiques sur l'une d'entre elles Passiflora quadrangularis L. Bull. Acad. Royal Scie. Bruxelles 9(1): 202-223.

Ramírez-B., W. 1970. Host specificity of fig wasps (Agaonidae). Evolution 24(4): 680-691.

Ramírez-B., W. 1986. Artificial hybridization and selffertilization in Ficus (Moraceae). Brenesia 25/26: 265-272.

Rieseberg, L.H. \& L. Brouillet. 1994. Are many species paraphyletic? Taxon 43: 21-32.

Sazima, M. \& I. Sazima. 1978. Bat-pollination of the Passion Flower, Passiflora mucronata, in Southeastern Brazil. Biotropica 10(2): 100-109.

Snow, A.A. 1982. Pollination intensity and potential seed set in Passiflora vitifolia. Oecologia 55(2): 231-237.

Snow, N. \& J.M. MacDougal. 1993. New chromosome reports in Passiflora (Passifloraceae). Syst. Bot. 18: 261-273.

Souza, M.M., G. Palomino, T.N.S. Pereira, M.G. Pereira \& A.P. Viana. 2004. Flow cytometric analysis of genome size variation in some Passiflora species. Hereditas 141: 31-38.

Stebbins, G.L. 1950. Variation and evolution in plants. 
Columbia Univ. Press, New York. 643 p. Vanderplank, J. 1996. Passion Flowers. $2^{\text {nd }}$. ed. The MIT Press, Massachusetts. 224 p.

Vanderplank, J. 2006. Passiflora miniata. Curtis's Bot. Mag. 23(3): 223-231.
Woodson, R.E. Jr. 1954. The North American species of Asclepias. Ann. Missouri Bot. Gard. 41: 1-211.

Wyatt, R. \& S.B. Broyles. 1994. Ecology and evolution of reproduction in milkweeds. Ann. Rev. Ecol. System. 25: 423-441. 


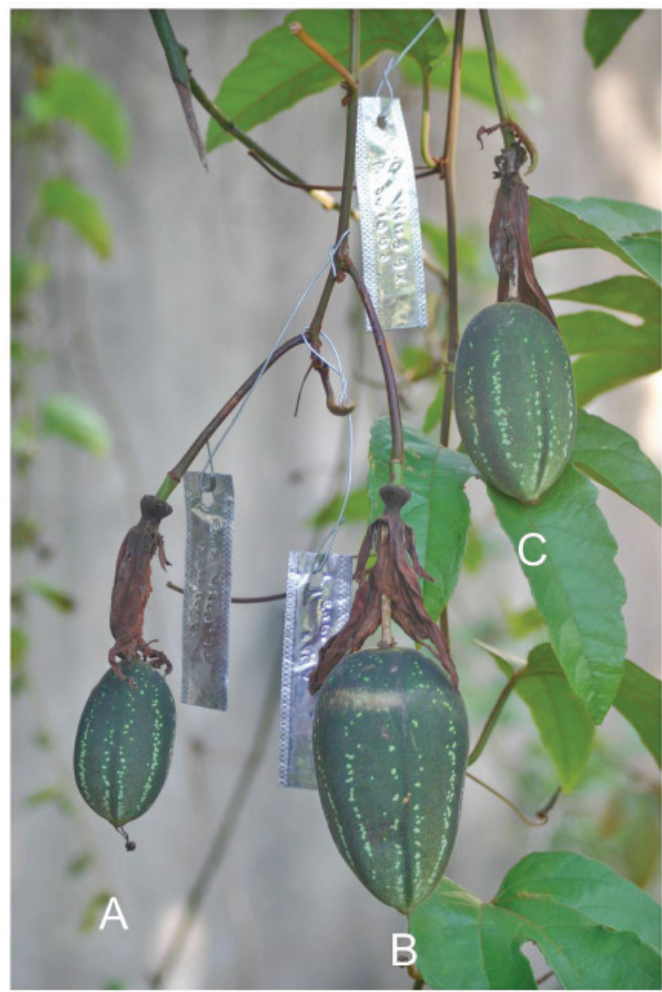

Fig. 1. Frutos de Passiflora vitifolia producto de la polinización cruzada con: A: P. platyloba; B: P. miniata y C: P. ambigua.

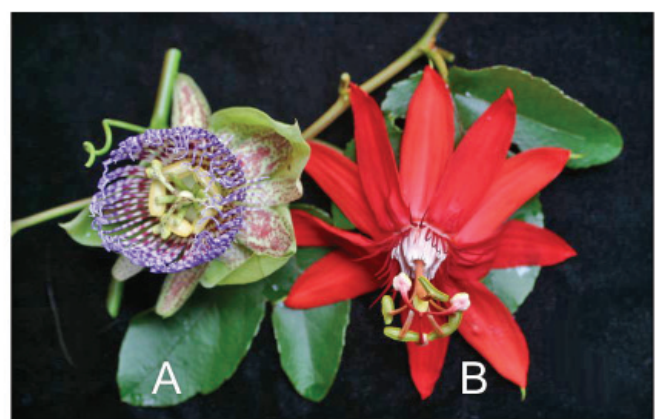

Fig. 4. A. Flor de Passiflora quadrangularis; características asociadas con polinización por abejas solitarias y avispas (ej. Scoliidae). B. Flor de $P$. vitifolia; características asociadas con polinización por colibríes.

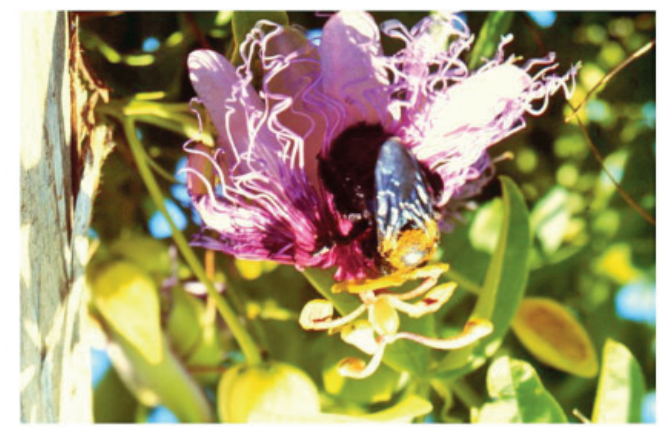

Fig. 6. Xylocopa sp. polinizando flor de maracuyá (P. edulisf. flavicarpa).

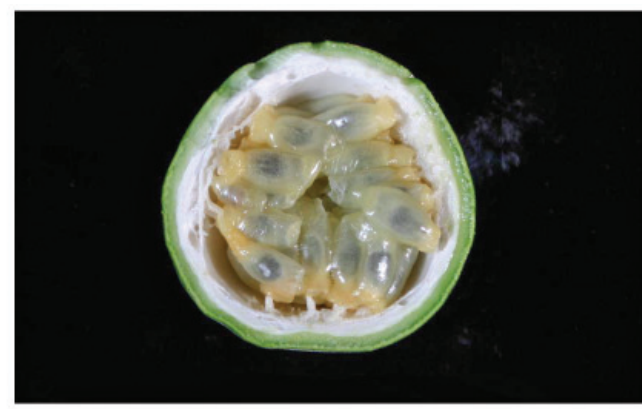

Fig. 2. Fruto de Passiflora vitifolia con semillas maduras del cruce con P. quadrangularis.

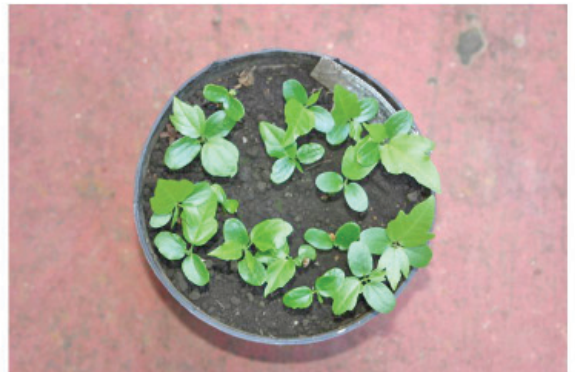

Fig. 3. Plantas híbridas de semillas del cruce de Passiflora vitifolia con P. ambigua (germinación 100\%).

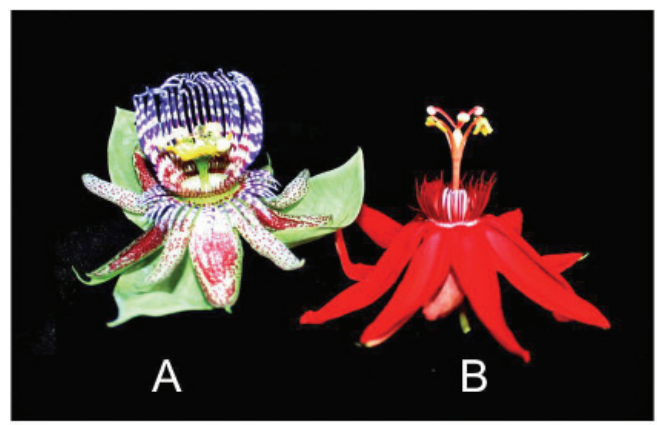

Fig. 5. A. Flor de Passiflora platyloba; corona parcialmente cortada para mostrar posición del androginóforo. B. Flor de P. vitifolia con androginóforo largo y expuesto.

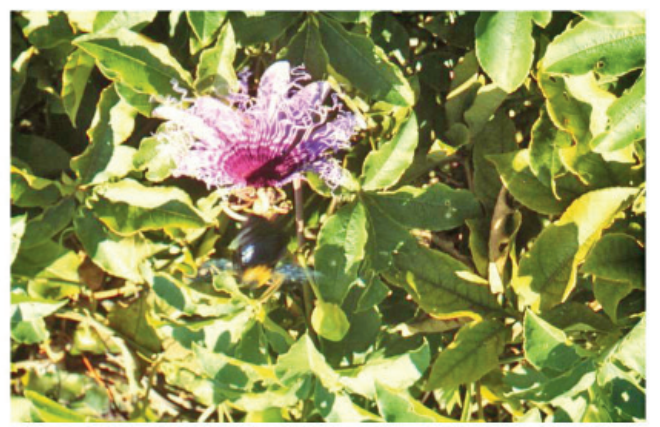

Fig. 7. Scoliidae sp. polinizando flor de maracuyá (P. edulis f. flavicarpa). 
